\title{
O Impacto da Pós-Graduação Stricto Sensu sobre o Estado de Saúde Mental do Brasileiro
}

The Impact of Postgraduate Studies on the Mental Health Status of Brazilians

Barbara Sant'ana Kuenka ${ }^{a}$

\begin{abstract}
Resumo: O presente estudo investigou o impacto dos cursos de pós-graduação stricto sensu sobre o estado de saúde mental do acadêmico brasileiro. Aplicou-se a metodologia Propensity Score Matching a informações da Pesquisa Nacional de Saúde (PNS), utilizando o diagnóstico de depressão clínica como proxy para o estado de saúde mental das observações. Resultados confirmaram o efeito depreciativo e estatisticamente relevante dos cursos de pós-graduação stricto sensu sobre o estado de saúde mental dos indivíduos da amostra analisada, especialmente sobre os homens. Estes achados refletem a necessidade de medidas de prevenção e incentivo do debate sobre saúde mental no círculo acadêmico.
\end{abstract}

Palavras-chave: Avaliação de Impacto; Saúde Mental; Propensity Score Matching. Classificação JEL: I21; I23; I26.

\begin{abstract}
The present study investigated the impact of graduate and postgraduate studies on the mental health status of Brazilian academics. The Propensity Score Matching methodology was applied to information from the Brazilian National Health Survey and the diagnosis of clinical depression was used as proxy for the mental health state of the observations. Results confirm the derogatory and statistically relevant effect of graduate and postgraduate studies on the mental health status of individuals in the sample analyzed, especially on men. These findings reflect the need for preventive measures and encouragement of the debate on mental health within the academic environment.
\end{abstract}

Keywords: Impact Evaluation; Mental Health; Propensity Score Matching. JEL Classification: I21; I23; I26.

\footnotetext{
${ }^{\text {a }}$ Mestre em Teoria Econômica pelo Programa de Pós-Graduação em Ciências Econômicas da Universidade Estadual de Maringá (PCE/UEM). E-mail: barbarakuenka@ gmail.com.
} 


\section{Introdução}

A pós-graduação stricto sensu vem apresentando crescimento contínuo no Brasil, registrando, somente entre 2010 e 2018, um aumento de $39 \%$ da sua quantidade de alunos matriculados (MARÉS, 2019; COORDENAÇÃO DE APERFEIÇOAMENTO DE PESSOAL DE NÍVEL SUPERIOR, 2018; 2019a; 2019b). De maneira geral, cursos de pós-graduação stricto sensu referem-se a programas de mestrado (acadêmico) e doutorado abertos a quaisquer indivíduos diplomados em algum curso superior de graduação. Não incluem, portanto, cursos como os de mestrado profissional e especialização, classificados como programas lato sensu (BRASIL, 2018).

Neste cenário, conjectura-se que esta tendência de ampliação do público pósgraduando esteja ligada a uma combinação de fatores, tais quais a expansão da oferta de cursos de pós-graduação via investimento e estímulos estatais; a maior pressão da sociedade e do mercado por níveis de escolarização mais elevados; e, sobretudo, ao avanço do desemprego - resultado da estagnação econômica brasileira na segunda metade do atual decênio (CIRANI et al., 2015). Entretanto, os efeitos característicos de uma conjuntura econômica desfavorável acabam por ultrapassar a barreira de indicadores estritamente socioeconômicos, podendo também afetar o estado de saúde psicoemocional da população. Evidências empíricas identificam um padrão de relacionamento entre desvantagens socioeconômicas e a prevalência de transtornos mentais nos indivíduos, especialmente entre jovens adultos (KNAPP et al., 2005; SANTOS; KASSOUF, 2007; ELSTAD; PEDERSEN, 2012; JAEN-VARAS et al., 2019).

Do ponto de vista socioeconômico, a relação entre saúde mental e pobreza se dá em um círculo vicioso multidimensional, envolvendo transtornos mentais, produtividade e emprego. A depressão mental - um dos distúrbios mentais mais prevalentes no mundo pode causar deficiências como inabilidade de estudar ou trabalhar, de forma que o não tratamento da doença resulta em episódios de graus cada vez mais severos, surtindo efeitos negativos sobre a produtividade do trabalho e, consequentemente, sobre o rendimento. A maior privação de recursos econômicos, por sua vez, constitui-se em um entrave para que este indivíduo despenda parte da sua renda no tratamento adequado dando, assim, continuidade a esse ciclo (WORLD HEALH ORGANIZATION, 2001).

Mais de 300 milhões de pessoas no planeta sofrem de depressão - a doença que mais incapacita pessoas no mundo (WORLD HEALTH ORGANIZATION, 2018; FANG et al., 2020). Segundo relatório da Organização Mundial da Saúde (WHO) divulgado em 2017, o Brasil apresenta a maior taxa de indivíduos que sofrem de depressão na América Latina (5,9\% da população), enquanto o suicídio ocupa a segunda maior causa de morte entre brasileiros de 15 a 29 anos (GLOBAL HEALTH INTELLIGENCE, 2017). Diante da evolução da ciência e da psicologia nesse plano, as discussões em relação às possíveis causas dos transtornos de ansiedade e depressão foram sendo ampliadas, de maneira a direcionar o debate para o quanto o sistema educacional e a busca pela alta qualificação podem afetar a saúde mental dos cidadãos. De fato, estudos científicos passaram a identificar que uma quantidade cada vez mais expressiva de universitários (na graduação 
e, principalmente, na pós-graduação) vem demonstrando sintomas de estresse, ansiedade ou depressão clínica (ADEWUYA et al., 2006; VÁZQUEZ; BLANCO, 2010; PELUSO et al., 2011; IBRAHIM et al., 2013; EVANS et al., 2018; COSTA; NEBEL, 2018; FOND et al., 2019).

Dessa forma, em virtude do crescimento do número de pós-graduandos brasileiros, da alarmante taxa de prevalência da doença no Brasil, dos recentes debates que sugerem a conexão entre ensino superior e piora dos níveis de estresse, ansiedade e depressão, bem como a aparente escassez de métodos de prevenção contra transtornos mentais no meio acadêmico, o presente trabalho tem como objetivo avaliar o impacto dos cursos de pósgraduação stricto sensu sobre o estado de saúde mental dos acadêmicos. Para isso, aplicou-se a metodologia Propensity Score Matching de avaliação de impacto a informações da Pesquisa Nacional de Saúde (PNS) do ano de 2013, a única publicada até a finalização deste artigo.

\section{O Panorama Teórico e Empírico}

Embora os sintomas variem de forma substancial entre indivíduos, transtornos mentais geralmente se caracterizam pela combinação de pensamentos, emoções e comportamentos anormais - esquizofrenia, depressão, transtorno de ansiedade, transtorno bipolar e abuso de substâncias são algumas das doenças que compõem essa complexa gama (MEIER et al., 2018; SALAGRE et al., 2018). Em um cenário típico de prevalência de depressão mental, o indivíduo apresenta oscilações de humor, redução de energia e queda de rendimento ao realizar atividades cotidianas. A capacidade de se concentrar e demonstrar interesse é reduzida, bem como manifestações de cansaço após qualquer tipo de esforço mínimo se mostram comuns (ZIMMERMAN et al., 2018). Problemas de autoestima e autoconfiança tendem a se juntar a sentimentos de culpa e ineptitude (WORLD HEALH ORGANIZATION, 2001).

Quanto ao embasamento empírico utilizado, esta seção elenca os trabalhos que de alguma forma se relacionam com a proposta aqui apresentada, iniciando com o bloco de estudos focados em amostras generalizadas de indivíduos, seguidas de estudos focados especificamente no público de pós-graduandos. Em cada bloco, os trabalhos estão organizados de forma que os nacionais sucedam aos internacionais, todos apresentados por ordem de publicação. A sistematização resumida da literatura pode ser observada no Quadro 1.

O debate acerca dos retornos da educação sobre a saúde dos indivíduos tem assumido crescente relevância nas últimas décadas, dentro e fora do arcabouço científico de Economia da Saúde. Nesse contexto, Feinstein (2002) apresenta evidências em relação aos impactos positivos que a educação gera sobre a saúde mental, embora de maneira geral - quando se busca fazer diagnósticos mais específicos para níveis superiores de educação - os efeitos evidenciados pela literatura passam a assumir formas negativas. $\mathrm{Na}$ Turquia, níveis de severidade moderados de depressão, ansiedade e estresse foram 
detectados entre jovens universitários de nível graduação, de forma que os escores mais altos se originaram do público feminino (BAYRAM; BILGEL, 2007).

Conforme estudos da área da saúde foram evidenciando as origens e os componentes da depressão - não apenas puramente biológicos, mas também sociais, econômicos e culturais - desenvolve-se, a posteriori, a preocupação com o relacionamento que a doença assume com elementos ambientais e comportamentais. Ao analisar os determinantes socioeconômicos da depressão mental para os brasileiros, Santos e Kassouf (2007) constataram que fatores como renda mensal per capita, estrutura familiar, idade e diferenças regionais exerciam efeito sobre a probabilidade de um indivíduo apresentar depressão clínica; sendo tais influências mais fortes, novamente, nas mulheres. Concomitantemente, quando trouxeram a análise para a esfera educacional, verificaram que quanto mais anos de estudo, menores as chances de se sofrer de depressão clínica.

Por outro lado, quando se observa especificamente o público de pós-graduandos, Hsu e Marshall (1987) haviam detectado, já na década de 1980, que a proporção de pósgraduandos com sintomas de depressão clínica era consideravelmente mais elevada que a do padrão geral da sociedade. Entretanto, a amostra observada incluía somente acadêmicos de Medicina, sendo que aqueles no primeiro ano da pós-graduação registraram os maiores escores de prevalência da doença. Também com o foco em pósgraduandos na área da saúde, Soriano et al. (2007) buscaram identificar a prevalência de Transtorno Afetivo Sazonal em meio a uma amostra de estudantes na Romênia. Segundo resultados, $11 \%$ da amostra de pós-graduandos sofriam de Transtorno Afetivo Sazonal no inverno e $2 \%$ no verão, concluindo que, assim como estudos similares para outras regiões, os indícios desse transtorno tendem a ser mais prevalentes no inverno do que nas outras estações.

Resultados parecidos são encontrados para grupos amostrais de outras localidades, destacando o caráter alarmante e crescente da prevalência de transtornos mentais em ambientes acadêmicos. Em análise exploratória da prevalência de depressão mental em estudantes universitários (graduação e pós-graduação), Ibrahim et al. (2013) identificaram e reuniram uma série de estudos publicados sobre a temática entre 1990 e 2010, e observaram taxas de prevalência em depressão que oscilavam de $10 \%$ a $85 \%$ das observações - em média, 30,6\% do conjunto examinado de acadêmicos do mundo todo apresentaram diagnóstico positivo de depressão mental no período.

Estendendo a análise também para outros distúrbios psicoemocionais, Al-Shayea (2014) identificou elevados escores de depressão, ansiedade e estresse entre pósgraduandos de diferentes programas em Ortodontia na Arábia Saudita. Acadêmicos casados e maiores de 30 anos de idade tendiam a apresentar prevalência menor em ansiedade quando comparados aos solteiros e mais jovens da amostra, indicando que iniciativas de apoio mental aos estudantes com estas características possam ser de grande utilidade em prevenir o alastramento das taxas de prevalência das patologias em questão. 


\section{Tabela 1 - Sistematização da Literatura Empírica}

\begin{tabular}{|c|c|c|c|c|c|}
\hline Autores & $\begin{array}{c}\text { Variáveis de } \\
\text { interesse }\end{array}$ & $\begin{array}{c}\text { Nível } \\
\text { geográfico }\end{array}$ & Período & Amostra & Metodologia \\
\hline \multicolumn{6}{|c|}{ Estudos internacionais e nacionais focados em estudantes de forma geral } \\
\hline $\begin{array}{l}\text { Feinstein } \\
\text { (2002) }\end{array}$ & $\begin{array}{l}\text { Depressão e } \\
\text { obesidade }\end{array}$ & $\begin{array}{l}\text { Internacional } \\
\text { (Grã-Bretanha) }\end{array}$ & 1999 e 2000 & $\begin{array}{l}\text { Adultos entre } 33 \text { e } \\
42 \text { anos de idade }\end{array}$ & $\begin{array}{c}\mathrm{MQO} \text { e } \\
\text { Propensity Score } \\
\text { Matching }\end{array}$ \\
\hline $\begin{array}{l}\text { Bayram; } \\
\text { Bilgel } \\
(2007) \\
\end{array}$ & $\begin{array}{c}\text { Depressão, } \\
\text { ansiedade, stress }\end{array}$ & $\begin{array}{l}\text { Internacional } \\
\text { (Turquia) }\end{array}$ & 2007 & $\begin{array}{l}1.617 \text { estudantes } \\
\text { de graduação }\end{array}$ & $\begin{array}{c}\text { Estatística } \\
\text { descritiva via } \\
\text { questionário }\end{array}$ \\
\hline $\begin{array}{l}\text { Santos; } \\
\text { Kassouf } \\
\text { (2007) }\end{array}$ & Depressão & Nacional & 2003 & $\begin{array}{l}158.098 \text { homens e } \\
\text { mulheres }\end{array}$ & $\begin{array}{l}\text { Regressões } \\
\text { probabilísticas }\end{array}$ \\
\hline \multicolumn{6}{|c|}{ Estudos internacionais e nacionais focados em estudantes de pós-graduação } \\
\hline $\begin{array}{c}\text { Hsu; } \\
\text { Marshall } \\
(1987) \\
\end{array}$ & Depressão & $\begin{array}{l}\text { Internacional } \\
\text { (Canadá) }\end{array}$ & 1984 & $\begin{array}{l}1.805 \text { estudantes } \\
\text { de pós-graduação } \\
\text { em Medicina }\end{array}$ & $\begin{array}{c}\text { Estatística } \\
\text { descritiva via } \\
\text { questionário }\end{array}$ \\
\hline $\begin{array}{l}\text { Soriano et } \\
\text { al. }(2007)\end{array}$ & $\begin{array}{c}\text { Prevalência de } \\
\text { Transtorno Afetivo } \\
\text { Sazonal } \\
\end{array}$ & $\begin{array}{l}\text { Internacional } \\
\text { (Romênia) }\end{array}$ & $\begin{array}{c}\text { Não } \\
\text { informado }\end{array}$ & $\begin{array}{l}476 \text { estudantes de } \\
\text { pós-graduação em } \\
\text { Medicina }\end{array}$ & $\begin{array}{c}\text { Análises de } \\
\text { variância e testes } \\
\text { chi-quadrado }\end{array}$ \\
\hline $\begin{array}{l}\text { Ibrahim et } \\
\text { al. (2013) }\end{array}$ & Depressão & Mundial & $\begin{array}{c}\text { Variável de } \\
\text { acordo com } \\
\text { localidade } \\
\end{array}$ & $\begin{array}{c}\text { Estudantes de } \\
\text { graduação e pós- } \\
\text { graduação } \\
\end{array}$ & Documental \\
\hline $\begin{array}{l}\text { Al-Shayea } \\
\text { (2014) }\end{array}$ & $\begin{array}{c}\text { Depressão, } \\
\text { ansiedade, stress }\end{array}$ & $\begin{array}{c}\text { Internacional } \\
\text { (Arábia } \\
\text { Saudita) } \\
\end{array}$ & 2013 & $\begin{array}{l}84 \text { estudantes de } \\
\text { pós-graduação em } \\
\text { Ortodontia }\end{array}$ & $\begin{array}{l}\text { Regressões } \\
\text { logísticas }\end{array}$ \\
\hline $\begin{array}{l}\text { Farrer et al. } \\
\qquad(2016)\end{array}$ & $\begin{array}{l}\text { Depressão e } \\
\text { ansiedade }\end{array}$ & $\begin{array}{l}\text { Internacional } \\
\text { (Austrália) }\end{array}$ & 2014 & $\begin{array}{l}611 \text { estudantes de } \\
\text { graduação e pós- } \\
\text { graduação }\end{array}$ & $\begin{array}{l}\text { Regressões } \\
\text { logísticas }\end{array}$ \\
\hline $\begin{array}{l}\text { Khushde et } \\
\text { al. (2017) }\end{array}$ & $\begin{array}{c}\text { Depressão, } \\
\text { homesickness e } \\
\text { vício em internet }\end{array}$ & $\begin{array}{l}\text { Internacional } \\
\text { (Irã) }\end{array}$ & 2015 e 2016 & $\begin{array}{l}204 \text { estudantes de } \\
\text { pós-graduação }\end{array}$ & $\begin{array}{c}\text { Estatística } \\
\text { descritiva via } \\
\text { questionário }\end{array}$ \\
\hline $\begin{array}{l}\text { Evans et al. } \\
\text { (2018) }\end{array}$ & $\begin{array}{l}\text { Depressão e } \\
\text { ansiedade }\end{array}$ & Mundial & $\begin{array}{l}\text { Variável de } \\
\text { acordo com } \\
\text { localidade }\end{array}$ & $\begin{array}{l}2.279 \text { estudantes } \\
\text { de pós-graduação }\end{array}$ & Documental \\
\hline $\begin{array}{l}\text { Fang et al. } \\
\text { (2019) }\end{array}$ & $\begin{array}{l}\text { Prevalência de } \\
\text { depressão e } \\
\text { ansiedade }\end{array}$ & $\begin{array}{l}\text { Internacional } \\
\text { (China) }\end{array}$ & 2018 & $\begin{array}{c}3.669 \text { estudantes } \\
\text { de pós-graduação } \\
\text { no } 1^{\circ} \text { ano do } \\
\text { mestrado }\end{array}$ & Machine learning \\
\hline $\begin{array}{l}\text { Souza et al. } \\
\quad(2018)\end{array}$ & $\begin{array}{l}\text { Síndrome de } \\
\text { Burnout }\end{array}$ & $\begin{array}{l}\text { Nacional } \\
\text { (Paraíba) }\end{array}$ & 2015 & $\begin{array}{c}\text { Estudantes de } \\
\text { mestrado em } \\
\text { Administração } \\
\end{array}$ & $\begin{array}{c}\text { Análise } \\
\text { qualitativa de } \\
\text { entrevistas } \\
\end{array}$ \\
\hline
\end{tabular}

Fonte: Elaboração própria (2021).

Os potenciais efeitos negativos da experiência universitária podem se tornar ainda mais prejudiciais quando combinados a fatores de risco de caráter socioeconômico e 
comportamental. Explorando outros fatores associados à prevalência de depressão clínica e transtorno generalizado de ansiedade, Farrer et al. (2016) verificaram que o risco de se tornar depressivo era maior para universitários pouco confiantes e insatisfeitos com a própria imagem corporal. Por outro lado, acadêmicos que passavam por dificuldades financeiras e sentiam pressão excessiva para se tornarem bem-sucedidos apresentaram maiores riscos de desenvolver Transtorno Generalizado de Ansiedade (TGA).

Ainda, no que se refere ao eixo interseccional contendo experiência universitária e características demográficas, Khushde et al. (2017) trazem à tona a influência que o deslocamento e distanciamento de casa desempenham sobre a plenitude psicoemocional dos pós-graduandos. Resultados para acadêmicos da Universidade de Sistan e Baluchistão, no Irã, evidenciaram que estudantes não nativos sofriam não apenas de níveis mais elevados de saudades do lar (homesickness), mas também de sintomas depressivos e vício pela internet. Para a China, por exemplo, os achados de Fang et al. (2019) ilustraram que 6,5\% de todos os pós-graduandos do país sofriam de depressão clínica. Já em escala mundial, Evans et al. (2018) verificaram que mestrandos e doutorandos exibiram taxas de prevalência em depressão e ansiedade seis vezes maiores que da população geral - no caso específico da depressão, concluíram que mais de um terço dos pós-graduandos detinham sintomas do transtorno.

No Brasil, o componente da saúde mental no ambiente universitário brasileiro é amplamente discutido na literatura científica de diversas áreas, contudo, poucos são os estudos dedicados apenas ao nicho específico de pós-graduandos. Nesse contexto, Souza et al. (2018) fizeram uma investigação de natureza qualitativa visando identificar possíveis indícios de estresse ocupacional crônico (Síndrome de Burnout) entre mestrandos da área de Administração. Por meio de entrevistas, os autores encontraram nos estudantes da amostra níveis significativamente elevados da síndrome, concluindo que parte do comportamento de risco para o desenvolvimento de tais patamares de estresse nasce dentro da própria academia.

Desta forma, a partir do levantamento empírico realizado, constatou-se que: a) a maioria dos trabalhos com a temática em questão se origina da área de saúde e biológicas (IBRAHIM et al., 2013; AL-SHAYEA, 2014; FARRER et al., 2016; KHUSHDE et al., 2017; EVANS et al., 2018) ou de encontros multidisciplinares (SORIANO et al., 2007; SOUZA et al., 2018; FANG et al, 2019); b) apenas dois dos estudos aqui citados advêm da área de Economia da Saúde (BAYRAM; BILGEL, 2007; SANTOS; KASSOUF, 2007), todavia, seus focos de análise não são voltados para o público de pós-graduandos; c) há uma lacuna na literatura de Economia da Saúde que se dedique à investigação do efeito que cursos de mestrado e doutorado possam trazer sobre o estado de saúde mental dos acadêmicos no Brasil, especialmente utilizando métodos de avaliação de impacto como ferramenta de análise.

\section{Estratégia Metodológica}

\subsection{Detalhamento das Variáveis}


Considerando a compatibilidade entre o objetivo do presente estudo e o conteúdo dos inquéritos disponíveis, optou-se pela utilização de micro dados por meio da retirada de informações da Pesquisa Nacional de Saúde (PNS) de 2013, seu único ano de publicação até o momento em que este estudo foi realizado. Trata-se de uma pesquisa em forma de questionário, realizada pelo Instituto Brasileiro de Geografia e Estatística (IBGE) em convênio com o Ministério da Saúde, que consiste na coleta domiciliar de informações referentes a estilo de vida, doenças crônicas, mercado de trabalho e percepção que os entrevistados têm do seu próprio estado de saúde. Os micro dados coletados são desagregados por sexo, grupos de idade, nível de instrução e cor ou raça para Brasil, grandes regiões e Unidades Federativas (BRASIL, 2014).

A variável de tratamento da análise, isto é, o fato de ter cursado ou não pósgraduação stricto sensu, assumiu o formato binário, tal que obteve valor 1 a observação que relatou já ter cursado um curso de mestrado ou a que relatou ter cursado mestrado e doutorado. Todas as observações restantes assumiram valor nulo. $\mathrm{O}$ detalhamento das variáveis pode ser observado na Tabela 2 .

$\mathrm{Na}$ ausência de qualquer outra pergunta presente no questionário da PNS que fizesse referência às características psicológicas dos indivíduos, utilizou-se o diagnóstico de depressão clínica como proxy para o estado de saúde mental dos entrevistados e, portanto, como variável de interesse na avaliação de impacto dos cursos de pós-graduação. Assim, assumiu valor unitário o entrevistado que tinha o diagnóstico positivo de depressão clínica e valor nulo o que não convivia com a patologia. Consequentemente, se o efeito do PSM tem sinal positivo, significa que a pósgraduação piora os níveis de depressão do indivíduo observado. Caso o sinal do efeito seja negativo, a pós-graduação estaria atuando como um fator de proteção contra a piora do estado de saúde mental das observações.

Apesar da escassez de dados oficiais sobre transtornos mentais no Brasil, destaca-se que o uso de diagnósticos profissionais em detrimento a variáveis auto percebidas - como no caso do presente estudo - contribui para reduzir a influência da subjetividade na quantificação das variáveis de saúde mental. Por outro lado, deve-se reconhecer que distúrbios psicoemocionais são patologias dotadas de significativo grau de subnotificação a nível mundial, de forma que a quantidade de diagnósticos formais nem sempre reflete a realidade da amostra analisada (ORGANIZAÇÃO PANAMERICANA DA SAÚDE, 2018; ELEFTHERIADES et al., 2020).

Já a escolha das demais variáveis provém do próprio arcabouço literário dos campos de psicologia e economia da saúde, vislumbrando controlar a estimação para diferenças de sexo, idade, raça e ocupação (GROSSMAN, 1972; KASSOUF, 1993; KENKEL, 1995; FAYISSA; TRAIAN, 2013). A fim de evitar problemas de incompatibilidade entre bases de dados, surge também a limitação de se trabalhar apenas com as informações disponibilizadas pelo questionário em questão, de forma que variáveis que poderiam ter importância no controle do impacto do tratamento acabaram não sendo incluídas. As variáveis de controle (sexo, idade, raça e ocupação) foram selecionadas de maneira a satisfazer a condição de balanceamento do modelo 
antes do pareamento dos escores, e o critério para a sua seleção foi o de que as variáveis de controle devem influenciar, simultaneamente, na participação junto ao tratamento (pós-graduação) e nos outcomes gerados (depressão clínica) (CUONG, 2012).

No que tange à diferenciação de sexo, esta se justifica pelas evidências discutidas na epidemiologia social de que a prevalência da depressão é aproximadamente o dobro sobre as mulheres em relação aos homens (RIECHER-RÖSSLER, 2010; WITTCHEN et al., 2011; BOYD et al., 2015; SASSARINI, 2016; KUEHNEN, 2017). Van de Velde, Bracke e Levecque (2010), por exemplo, confirmam esse indicativo de discrepância no estado de saúde mental dos gêneros em 23 países europeus - reforçando que a maior prevalência de transtornos mentais em meio ao público feminino consiste em uma tendência mundial.

Quanto à divisão por grupos de idade, Stordal, Mykletun e Dahl (2003) reforçam a relevância de se atentar para os diferentes níveis de prevalência entre distintos grupos de faixa etária, além de que indivíduos de idades diferentes podem sentir e enxergar os sintomas de maneira distinta (KNESPER et al., 1987; OHRNBERGER et al., 2017). Sob a ótica da produção em saúde, admite-se ainda que quanto mais elevada a idade dos indivíduos, mais depreciado tende a estar seu estoque de saúde em comparação aos mais jovens (GROSSMAN, 1972; FAYISSA; GUTEMA, 2006).

Ainda com base na literatura científica, optou-se por controlar para diferenças raciais mediante ao maior padrão de prevalência de transtornos psicoemocionais em meio a indivíduos não brancos. Grupos étnicos estão suscetíveis a distintas realidades socioeconômicas, estruturais e culturais. Estas que podem influenciar negativamente diferentes aspectos referentes à saúde mental individual: qualidade do sono, relacionamentos pessoais, satisfação corporal, hábitos alimentares, entre outros (JOHNS et al., 2017; ALAIE et al., 2018; CICIURKAITE; PERRY, 2018; HUANG et al., 2019).

Por fim, a ocupação é introduzida a fim de controlar a interferência que a participação no mercado de trabalho pode exercer sobre o estado de saúde mental dos entrevistados. A literatura mostra que o fato de estar empregado pode ser benéfico para a plenitude psicoemocional (BENJET et al., 2009). Além disso, a quantidade de recursos econômicos que um determinado indivíduo detém pode influenciar no seu estado de saúde, de forma que seja possível inferir que tais consequências se estendam para a saúde mental (MAGALHÂES, 1978; PHELPS, 2016). É comum encontrar uma ligação positiva entre renda e estado de saúde nos estudos dessa temática (NIXON; ULMANN, 2006; FOLLAND; GOODMAN; STANO, 2013). Entretanto, a especificação para o diagnóstico de depressão torna ambíguo o sinal esperado dessa relação - ao mesmo tempo em que, por exemplo, graus mais altos de produtividade ou maiores cargas horárias de trabalho são capazes de aumentar rendimentos, estas também podem provocar considerável desgaste psicológico.

A partir da composição total de 205.546 entrevistados pela PNS, foram eliminadas as observações que não responderam às perguntas selecionadas para compor as variáveis, além dos missings recorrentes de outros possíveis erros originados da base de dados. 
Após a limpeza do plano amostral, a amostra a ser utilizada para a estimação do PSM passou a ser composta por 50.103 observações, das quais 21.627 são do sexo masculino e 28.476 do sexo feminino.

Tabela 2 - Detalhamento das Variáveis Usadas, Pesquisa Nacional de Saúde (PNS), 2013

\begin{tabular}{|c|c|c|}
\hline Variável & Definição no questionário da PNS & Descrição \\
\hline \multicolumn{3}{|l|}{ Tratamento } \\
\hline Pós-graduação & $\begin{array}{l}\text { Qual foi o curso mais elevado que } \\
\text { frequentou anteriormente? }\end{array}$ & $\begin{array}{l}1 \text { para Mestrado/Doutorado; } \\
0 \text { para o restante }\end{array}$ \\
\hline \multicolumn{3}{|l|}{ Outcome } \\
\hline Depressão & $\begin{array}{l}\text { Algum médico ou profissional de } \\
\text { saúde mental (como psiquiatra ou } \\
\text { psicólogo) já lhe deu o diagnóstico } \\
\text { de depressão? }\end{array}$ & $\begin{array}{l}1 \text { para } \mathrm{Sim} ; \\
0 \text { para Não }\end{array}$ \\
\hline \multicolumn{3}{|l|}{ Controle } \\
\hline Sexo & Qual o seu sexo? & $\begin{array}{l}1 \text { para Feminino; } \\
0 \text { para Masculino }\end{array}$ \\
\hline Idade & Qual a sua idade? & $\begin{array}{l}1 \text { para Entre } 20 \text { e } 59 \text { anos; } \\
0 \text { para } 60 \text { anos ou mais }\end{array}$ \\
\hline Raça & Cor ou raça & $\begin{array}{l}1 \text { para Brancos; } \\
0 \text { para Pretos, Amarelos, } \\
\text { Pardos ou Indígenas (não } \\
\text { brancos) }\end{array}$ \\
\hline Ocupação & $\begin{array}{l}\text { Na semana de } 21 \text { a } 27 \text { de julho de } \\
2013 \text { (semana de referência), } \\
\text { trabalhou ou estagiou, durante pelo } \\
\text { menos uma hora, em alguma } \\
\text { atividade remunerada em dinheiro? }\end{array}$ & $\begin{array}{l}1 \text { para } \mathrm{Sim} ; \\
0 \text { para Não }\end{array}$ \\
\hline
\end{tabular}

Fonte: Elaboração própria (2021), com informações da Pesquisa Nacional de Saúde (2013).

\subsection{A Metodologia Propensity Score Matching (PSM)}

O pareamento por escore de propensão (Propensity Score Matching) constrói um grupo estatístico de comparação ao modelar a probabilidade de participar de um determinado programa com base nas características observáveis não afetadas pelo tratamento em si (KHANDER; KOOLWAL; SAMAD, 2010). Participantes são então pareados com não participantes a partir dessa probabilidade - denominada de escore de propensão.

Por meio das técnicas de pareamento, tenta-se criar um grupo de controle que seja o mais parecido possível com o grupo de tratamento no que se concerne a características observáveis. Em outras palavras, deve-se parear indivíduos "não tratados" que apresentem características não afetadas pelo programa similares às dos "tratados". Em 
seguida, cada indivíduo exposto ao tratamento é pareado com um contrafactual observacionalmente parecido, de maneira que a diferença média de resultados (outcomes) nos dois grupos é comparada com o objetivo de expressar o efeito da iniciativa, programa ou tratamento (ROSENBAUM; RUBIN, 1983; SOUZA, 2010; KHANDER; KOOLWAL; SAMAD, 2010).

Isso posto, adapta-se a metodologia PSM à proposta do presente estudo com o objetivo de (i) estimar a probabilidade de se cursar um programa de pós-graduação (tratamento) com base em algumas características dos indivíduos (variáveis de controle); (ii) encontrar, a partir dessa probabilidade, indivíduos na amostra que não cursaram pósgraduação, mas que apresentam atributos idênticos aos dos pós-graduados; e, finalmente, (iii) parear pós-graduados e não pós-graduados de características parecidas.

Assim, conforme Caliendo e Kopeinig (2005), o efeito de cursar pós-graduação é aqui representado pelo efeito médio do tratamento sobre os tratados (Average Treatment Effect on the Treated - ATT ou ATET), expresso por:

$$
\mathrm{ATT}=E\left[Y_{i}(1)-Y_{i}(0) \mid T_{i}=1\right]=E\left[Y_{i}(1) \mid T_{i}=1\right]-E\left[Y_{i}(0) \mid T_{i}=1\right]
$$

Sendo $\mathrm{T}_{\mathrm{i}}$ igual a 1 se o indivíduo pertence ao grupo dos tratados e igual a 0 se não participou da intervenção. A vantagem do uso do ATT em detrimento ao mais generalizado ATE (average treatment effect - efeito médio do tratamento) se dá pelo fato de este último inclui o efeito do programa sobre indivíduos para os quais a intervenção não foi criada - apresentando, assim, menor relevância para os desenvolvedores de políticas socioeconômicas. O ATT, por outro lado, foca inteiramente no efeito do tratamento sobre indivíduos que de fato estão propensos a participarem dele (HECKMAN; ICHIMURA; TODD, 1997; CALIENDO; KOPEINIG, 2005).

A estimação de um modelo de participação pode ser feita sob uma série de técnicas, geralmente por meio de um modelo probit ou logit. Nesse sentido, o ponto positivo está no uso de uma única base de dados para a estimação de um modelo probit, já que uma mesma fonte de dados para encontrar tratados e controle ajuda a assegurar que características observadas presentes no modelo probit sejam mensuradas similarmente nos dois grupos e, assim, reflitam os mesmos conceitos (KHANDER; KOOLWAL; SAMAD, 2010).

Uma vez estimada a equação de participação, é possível prever os outcomes que representam os escores de propensão - ou seja, a probabilidade estimada de se cursar pósgraduação. Nesse cenário, cada participante e não participante da amostra terá um escore estimado na forma $\hat{P}(X \mid T=1)=\hat{P}(X)$. A partir disso, a região de suporte comum é definida - isto é, as distribuições de escores de propensão de tratamento e grupo de controle se sobrepõem - e o pareamento entre pós-graduados (tratados) e não pósgraduados (contrafactuais) é realizado.

Diferentes abordagens com diferentes critérios são usadas com a finalidade de parear tratados e contrafactuais. Isso remete ao cálculo de um peso para cada conjunto participante-não participante que fora pareado com base nos escores de propensão - dessa 
forma, a escolha de uma determinada técnica pode afetar o resultado estimado do programa por conta dos pesos atribuídos a cada uma delas (ZANUTTO, 2006; AUSTIN et al., 2018). Para fins de robustez, foram três as técnicas aqui utilizadas: pareamento por vizinho mais próximo (nearest-neighbor matching), onde cada unidade de tratamento é pareada com uma unidade de comparação que tenha o escore de propensão mais próximo - pode-se escolher $n$ vizinhos mais próximos, embora comumente se use 5; radius matching, que através de um caliper visa reduzir a diferença entre escores de propensão de tratado e contrafactual e impõe um grau de tolerância na distância máxima entre as probabilidades; e kernel, o estimador de pareamento não paramétrico que utiliza uma média ponderada de todos os não participantes para construir o controle correspondente para cada participante (CALIENDO; KOPEINIG, 2005). Os pesos do pareamento por kernel são dados por:

$$
w(i, j)_{K M}=\frac{K\left(\frac{P_{j}-P_{i}}{a_{n}}\right)}{\sum_{k \in C} K\left(\frac{P_{k}-P_{i}}{a_{n}}\right)}
$$

onde $P_{i}$ é o escore de propensão do participante $i, P_{j}$ o escore do não participante $j$, $K(\cdot)$ é uma função kernel e $a_{n}$ uma banda (bandwidth) de parâmetro. O efeito médio do tratamento é igual à diferença média em outcomes na área de suporte comum, ponderando as unidades de comparação pela distribuição de escores de propensão dos pós-graduados (KHANDER; KOOLWAL; SAMAD, 2010).

No que se refere à seleção das covariáveis - isto é, o conjunto de características observáveis no PSM - Caliendo e Kopeinig (2005) reforçam que se deve optar apenas por variáveis de controle que influenciam, ao mesmo tempo, os outcomes e a decisão de participar do tratamento. Em última instância, as covariáveis devem obedecer ao requisito teórico da CIA, que permite admitir que as covariáveis selecionadas não são afetadas pela intervenção sendo analisada. Para assegurar que isso ocorra, a recomendação é de que as covariáveis sejam fixadas no tempo (HECKMAN; ICHIMURA; TODD, 1997). O fato de o presente estudo consistir em uma análise de corte transversal facilita que este requisito seja satisfeito.

\section{Resultados e Discussão}

A amostra disponibilizada pela Pesquisa Nacional de Saúde contou, na sua totalidade, com 205.546 indivíduos entrevistados, dos quais 1.014 alegaram ter frequentado cursos de pós-graduação stricto sensu (mestrado ou doutorado). Esta parcela representa $0,49 \%$ da amostra observada, o que reflete os percentuais brasileiros relativamente baixos em relação ao resto do mundo, quando se trata do número de pósgraduados na sua população (GIORDAN, 2019; ORGANISATION FOR ECONOMIC CO-OPERATION AND DEVELOPMENT, 2019). A estatística descritiva da amostra é exibida na Tabela 3. 
A partir das variáveis selecionadas para o estudo, observou-se que, na amostra completa da PNS, as mulheres consistiram na maioria entrevistada $(51,72 \%$ da amostra contra 48,28\% dos homens). No que se refere ao grupo caracterizado por ter frequentado cursos de pós-graduação, o sexo feminino teve pequena vantagem $(0,25 \%)$ em relação aos homens $(0,24 \%)$ - verificando-se, portanto, pouca diferença nos percentuais registrados por homens e mulheres pós-graduados.

Dentre os grupos etários, a maioria presente da amostra consistiu em indivíduos entre 20 e 59 anos (55,58\%), ao passo que aqueles maiores de 60 anos de idade representaram $44,42 \%$ dos entrevistados - $0,41 \%$ das pessoas que alegaram ter frequentado um programa de mestrado ou doutorado tinham entre 20 e 59 anos de idade, enquanto $0,08 \%$ desse mesmo grupo de pós-graduados eram maiores de 60 anos no momento em que a pesquisa fora realizada. Esta diferença nos percentuais de grupos etários pode ser interpretada como um indicativo que reforça como o ingresso do brasileiro na pós-graduação stricto sensu tem assumido tendência de crescimento nas últimas décadas (MARÉS, 2019).

Tabela 3 - Perfil Descritivo da Amostra, Pesquisa Nacional de Saúde (PNS), 2013

\begin{tabular}{|c|c|c|c|}
\hline Variável & $\begin{array}{c}\text { Cursou pós- } \\
\text { graduação } \\
(\mathrm{n}=1.014) \\
(\%)\end{array}$ & $\begin{array}{c}\text { Não cursou pós- } \\
\text { graduação } \\
(\mathbf{n}=\mathbf{2 0 4 . 5 3 2}) \\
(\%)\end{array}$ & $\begin{array}{c}\text { Total } \\
(\mathrm{n}=\mathbf{2 0 5 . 5 4 6 )} \\
(\%)\end{array}$ \\
\hline \multicolumn{4}{|l|}{ Sexo } \\
\hline Sexo feminino & 0,25 & 51,47 & 51,72 \\
\hline Sexo masculino & 0,24 & 48,03 & 48,28 \\
\hline \multicolumn{4}{|l|}{ Idade } \\
\hline Idade entre 20 e 59 anos & 0,41 & 55,18 & 55,58 \\
\hline Idade maior que 60 anos & 0,08 & 44,33 & 44,42 \\
\hline \multicolumn{4}{|l|}{ Raça } \\
\hline Brancos & 0,35 & 37,68 & 38,03 \\
\hline Não brancos & 0,14 & 61,83 & 61,97 \\
\hline \multicolumn{4}{|l|}{ Ocupação } \\
\hline Ocupados & 0,40 & 41,54 & 41,94 \\
\hline Não ocupados & 0,09 & 57,97 & 58,06 \\
\hline \multicolumn{4}{|l|}{ Saúde mental } \\
\hline Tem depressão & 0,02 & 2,04 & 2,06 \\
\hline Não tem depressão & 0,47 & 97,47 & 97,94 \\
\hline
\end{tabular}

Fonte: Elaboração própria (2021) com informações da Pesquisa Nacional de Saúde (2013).

Nota: O percentual para cada variável é calculado com base no total de observações da amostra completa ( $\mathrm{n}=205.546$ indivíduos).

Em relação ao quesito racial, $38,03 \%$ da amostra total da PNS correspondeu a entrevistados brancos, evidenciando uma maioria de $61,97 \%$ de entrevistados pretos, pardos, amarelos ou indígenas. Ademais, dentre os $0,49 \%$ que alegaram frequentar cursos de pós-graduação, $0,35 \%$ eram brancos, contra $0,14 \%$ não brancos. 
Ilustrando a participação dos entrevistados no mercado de trabalho, observa-se que uma maioria de 58,06\% respondeu não estar ocupada, enquanto 41,94\% alegaram ter trabalhado ou estagiado por remuneração em dinheiro na semana em que a pesquisa foi realizada. Por outro lado, quando o foco se volta para o grupo específico de pósgraduados, a parcela de indivíduos ocupados passa a ser maior que a de desocupados (0,40\% pós-graduados ocupados contra $0,09 \%$ pós-graduados desocupados), sugerindo que maiores níveis de qualificação educacional tendem a abrir mais portas no mercado de trabalho (CIRANI et al., 2015).

Finalmente, no que se refere à variável de interesse aqui observada, verificou-se uma taxa de prevalência de depressão clínica de 2,06\% dentro da amostra geral da PNS. Já dos $0,49 \%$ que cursaram pós-graduação, $0,02 \%$ alegaram ter recebido o diagnóstico positivo para depressão clínica - sendo considerados, na presente pesquisa, como indivíduos de saúde mental deteriorada.

Após a devida seleção e limpeza da amostra, aplicou-se a metodologia Propensity Score Matching (PSM) para avaliar qual foi o impacto da pós-graduação stricto sensu sobre o estado de saúde mental - medido pelo diagnóstico de depressão - dos que alegaram ter frequentado cursos de mestrado ou doutorado. Desta forma, a amostra analisada passou a ser de 50.103 indivíduos, dentre os quais 487 - isto é, menos de $1 \%$ cursaram pós-graduação (grupo de tratados), enquanto as 49.616 observações restantes assumiram o grupo de controle.

\section{Tabela 4 - Efeitos da Pós-Graduação Stricto Sensu sobre o Estado de Saúde Mental dos Acadêmicos por Propensity Score Matching}

\begin{tabular}{|c|c|c|c|}
\hline \multirow{2}{*}{$\begin{array}{l}\text { Variável de interesse } \\
\text { (outcome) }\end{array}$} & \multicolumn{3}{|c|}{$\begin{array}{l}\text { Efeito médio do tratamento sobre os tratados (ATT) e } \\
\text { significância }\end{array}$} \\
\hline & Geral & Homem & Mulher \\
\hline \multicolumn{4}{|l|}{ Saúde mental } \\
\hline Nearest neighbor & $0,017 *$ & $0,016^{*}$ & 0,018 \\
\hline Radius matching $\mathbf{0 , 1}$ & 0,017 * & $0,018 *$ & 0,025 \\
\hline Radius matching $\mathbf{0 , 0 1}$ & $0,017 * * *$ & 0,017 & 0,024 \\
\hline Kernel matching & $0,017 *$ & 0,018 & 0,025 \\
\hline n tratados & 487 & 244 & 243 \\
\hline n controle & 49.616 & 21.383 & 28.233 \\
\hline n blocos & 5 & 4 & 5 \\
\hline \multicolumn{4}{|l|}{ Variáveis de controle } \\
\hline \multicolumn{4}{|l|}{ Sexo } \\
\hline \multicolumn{4}{|l|}{ Idade } \\
\hline \multicolumn{4}{|l|}{ Raça } \\
\hline Ocupação & & & \\
\hline
\end{tabular}

Fonte: Elaboração própria (2020).

Nota: ***Significativo a $1 \%$. **Significativo a $5 \%$. *Significativo a $10 \%$. 
Conforme mencionado anteriormente, as estimações foram realizadas sob três diferentes técnicas de pareamento - nearest neighbor (vizinho mais próximo), radius matching (calibres 0,1 e 0,01) e kernel (banda 0,06). Buscando evitar a estimação incorreta dos erros padrões, decidiu-se pelo uso do bootstrapping, técnica pela qual amostras repetidas são extraídas da amostra original para que o erro padrão da estimativa seja novamente estimado com cada amostra. Considerando que os estimadores são assintoticamente lineares, o bootstrapping tende a levar a erros padrões e intervalos de confiança válidos (KHANDER; KOOLWAL; SAMAD, 2010). Após controlar a análise para as variáveis sexo, idade, raça e ocupação, a propriedade de equilíbrio do modelo foi satisfeita. Os resultados são expostos na Tabela 4. Os resultados da estimação do probit pela qual passa o processo de pareamento podem ser observados na Tabela 1 do Apêndice, ao final deste trabalho.

Os efeitos da pós-graduação sobre o estado de saúde mental de toda a amostra observada estão apresentados na coluna denominada "geral". Buscando verificar se os efeitos foram influenciados pela predominância de um dos sexos, aplicou-se a mesma análise separadamente para homens e mulheres - cujos resultados completam as duas colunas à direita. Novamente, é oportuno mencionar que, considerando como as variáveis foram construídas, sinais positivos remeteriam a uma relação positiva entre pósgraduação stricto sensu e o diagnóstico positivo de depressão clínica; isto é, depreciação do estado de saúde mental. Sinais negativos, por outro lado, sugeririam que a pósgraduação tenha atuado como um meio de proteção à saúde mental dos indivíduos.

A partir dos resultados (Tabela 4), observou-se o efeito prejudicial da pósgraduação sobre a saúde mental do grupo "geral", significativo em todas as técnicas de pareamento utilizadas. Neste cenário, a estimação dos erros por meio do bootstrapping permite que a leitura do efeito ATT seja feita a partir dos efeitos marginais (STATACORP, 2013): participar da pós-graduação stricto sensu aumenta em 0,017 a probabilidade de se obter um diagnóstico positivo para depressão clínica. No caso dos homens, em específico, esse aumento é de 0,016 (nearest neighbor) a 0,018 (radius). A aplicação do bootstrapping na estimação dos efeitos de tratamentos se dá na criação de uma espécie de subamostra, retirada da amostra original pareada. Dessa amostra substituta, estima-se o desvio padrão dos efeitos de tratamento - que será usado como estimativa do erro padrão do efeito do tratamento na amostra original em si (AUSTIN; SMALL, 2014). Seu uso se justifica por se tratar de uma técnica que oferece estimativas mais precisas do que as geradas a partir da amostra original (NISBET, 2018).

A Tabela 5, por sua vez, traz os resultados dos testes que verificaram a qualidade dos pareamentos realizados. Nas três amostras estudadas (geral, homens e mulheres), a média do viés foi reduzida a zero após o pareamento, assim como os valores de B (Rubin's B) e R (Rubin's R). B consiste na diferença das médias dos índices lineares de escores de propensão nos grupos de tratados e não tratados e, quando abaixo de $25 \%$, indica um grupo de controle devidamente balanceado. $\mathrm{O}$ valor $\mathrm{R}$ - que por recomendação deve estar dentro do intervalo entre 0,5 e 2 - expressa a razão das variâncias dos escores de tratados e controle (RUBIN, 2001). Ainda, conforme Hagen (2016), um pseudo-R ${ }^{2}$ 
nulo também indica que as diferenças entre pós-graduados e não pós-graduados foram devidamente balanceadas. Em outras palavras, admite-se que os pressupostos para um modelo balanceado (CIA) foram atendidos e que o pareamento é estatisticamente apropriado. Os testes de balanceamento para cada covariável são apresentados na Tabela 2 do Apêndice.

Voltando aos resultados apresentados na Tabela 4, é possível verificar que tais achados são consistentemente corroborados pela literatura a respeito do tema. Evidências confirmam o relacionamento direto entre cursos de mestrado e doutorado e transtornos de ansiedade, Transtorno Afetivo Sazonal, estresse excessivo e, como no caso do presente estudo, depressão clínica (HSU; MARSHALL, 1987; SORIANO et al., 2007; IBRAHIM et al., 2013; AL-SHAYEA, 2014; FARRER et al., 2016; EVANS et al, 2018; FANG et al., 2019).

\section{Tabela 5 - Teste de Balanceamento (redução de viés) para Verificar a Qualidade do} Pareamento

\begin{tabular}{l|c|c|c|c|c}
\hline \hline $\begin{array}{l}\text { Amostra "geral" } \\
\text { antes e depois do } \\
\text { pareamento }\end{array}$ & Pseudo R & $\begin{array}{l}\text { Chi-quadrado } \\
\text { e significância }\end{array}$ & $\begin{array}{c}\text { Média } \\
\text { do viés }\end{array}$ & B (\%) & $\mathbf{R}$ \\
\hline \multicolumn{1}{c|}{ Antes } & 0,063 & $348,100^{* * *}$ & 36,2 & 90,2 & 0,69 \\
\hline \multicolumn{1}{c|}{ Depois } & 0,000 & 0,000 & 0,0 & 0,0 & 1,0 \\
\hline $\begin{array}{l}\text { Amostra "homem" } \\
\text { antes e depois do } \\
\text { pareamento }\end{array}$ & Pseudo R & $\begin{array}{l}\text { Chi-quadrado } \\
\text { e significância }\end{array}$ & $\begin{array}{c}\text { Média } \\
\text { do viés }\end{array}$ & $\mathbf{B}(\%)$ & $\mathbf{R}$ \\
\hline \multicolumn{1}{c|}{ Antes } & 0,046 & $124,050^{* * *}$ & 33,5 & 74,7 & 0,83 \\
\hline \multicolumn{1}{c|}{ Depois } & 0,000 & 0,000 & 0,0 & 0,0 & 1,0 \\
\hline $\begin{array}{l}\text { Amostra "mulher" } \\
\text { antes e depois do } \\
\text { pareamento }\end{array}$ & Pseudo R & $\begin{array}{l}\text { Chi-quadrado } \\
\text { e significância }\end{array}$ & $\begin{array}{c}\text { Média } \\
\text { do viés }\end{array}$ & $\mathbf{B}(\%)$ & $\mathbf{R}$ \\
\hline \multicolumn{1}{c|}{ Antes } & 0,080 & $223,360^{* * *}$ & 52,5 & 104,1 & 0,65 \\
\hline \multicolumn{1}{c|}{ Depois } & 0,000 & 0,000 & 0,0 & 0,0 & 1,0 \\
\hline \hline
\end{tabular}

Fonte: Elaboração própria (2020).

Nota: $* * *$ Significativo a $1 \%$.

A crescente piora dos indicadores de saúde mental dos pós-graduandos é atribuída a uma variada gama de fatores. A cultura acadêmica tem se alastrado em todo o mundo e, com isso, forma indivíduos altamente qualificados a uma quantidade e velocidade cada vez mais elevadas, de maneira que o ritmo de crescimento do número de pós-graduados formados excede ao ritmo de crescimento da capacidade de absorção do próprio mercado (BISHOP, 1993; COUGHLAN, 2015). Esta redução das oportunidades de emprego acaba por inibir a independência financeira dos acadêmicos, provocando elevações de estresse e 
sentimentos de angústia quando confrontados com suas opções no médio e no longo prazo (ELEFTHERIADES et al., 2020).

Ainda, estudantes de pós-graduação stricto sensu lidam com a crescente competição não somente nos processos seletivos para os cursos, mas também para manter sua vaga, seu desempenho na produção de artigos científicos e, em muitos casos, suas bolsas de estudo. Segundo informações para uma amostra de 2.903 estudantes de mestrado e doutorado de todo o Brasil, Costa e Nebel (2018) constataram que $45 \%$ dos pós-graduandos que entrevistaram se sentiam excessivamente cobrados pelo seu orientador ou programa para publicar artigos em periódicos. Além disso, $40 \%$ alegaram ter como maior preocupação o medo de perder o emprego ou a bolsa de estudos, seguida pelo medo de não terminar a tese ou dissertação (37\%).

Cursar um programa acadêmico desta natureza requer, muitas vezes, dedicação em tempo integral, tal que a opção de cursar uma pós-graduação pode frequentemente se tornar um tradeoff entre atingir um nível mais alto de qualificação e ocupar ativamente o mercado de trabalho. No Brasil, cerca de 70\% dos pós-graduandos não exercem nenhuma outra atividade profissional além da pós-graduação em si. Por outro lado, $30 \%$ vivem com o(a) cônjuge e/ou filhos (COSTA; NEBEL, 2018): o que constitui um potencial indicativo para o porquê de as preocupações com emprego e renda futura estarem presentes entre as causas para o sofrimento psicológico estudantil (LEVECQUE et al., 2017).

Por outro lado, os resultados da análise por diferenciação de sexo (Tabela 4) sugerem uma influência do público masculino nas estimações do grupo geral, uma vez que os efeitos dos cursos de mestrado e doutorado foram nocivos para a plenitude psicoemocional dos homens, ao passo que, para as mulheres, não tiveram significância estatística. Esta constatação é inesperada, dada a frequência com que o público feminino figura como o sexo mais suscetível ao desenvolvimento de transtornos mentais (RIECHER-RÖSSLER, 2010; VAN DE VELDE; BRACKE; LEVECQUE, 2010; WITTCHEN et al., 2011; BOYD et al., 2015; SASSARINI, 2016; KUEHNEN, 2017). Em contrapartida, destaca-se que a diferenciação de sexo pode ter apresentado quantidade menor de resultados significativos por conta da redução de tamanho das amostras quando foram divididas em grupos masculino e feminino, deixando o número de tratados relativamente pequeno para as estimações.

Como possível explicação para o grupo masculino ter aqui apresentado maior suscetibilidade à deterioração psicoemocional, poder-se-ia inferir que homens possam se sentir mais pressionados a ocuparem e exercerem o papel de chefes e provedores da família. Isso, pois, no ano de 2013 (quando a PNS foi publicada), 61,2\% das famílias eram chefiadas por homens, enquanto os $38,8 \%$ restantes tinham mulheres como principal provedora (BRASIL, 2015). Já para as mulheres, o próprio fato de terem cursado pósgraduação pode explicar o porquê de os efeitos não terem sido significativos, uma vez que níveis educacionais mais baixos consistem em um dos principais fatores socioeconômicos de risco para as taxas de prevalência da depressão clínica em meio ao sexo feminino (VAN DER WAERDEN et al., 2014). Diante das discrepâncias de gêneros aqui encontradas - identificando no homem pós-graduado a maior tendência para o 
sofrimento mental - atenta-se para as potenciais consequências do não tratamento de um distúrbio como o da depressão clínica: homens com alto nível de qualificação acadêmica são aproximadamente três vezes mais inclinados a cometerem suicídio em comparação às mulheres em mesma situação (ELEFTHERIADES et al., 2020).

Proporcionada pela evolução do diálogo sobre saúde mental nas últimas décadas, a redução do preconceito acerca das patologias psicoemocionais simboliza um respeitável avanço. Entretanto, a estigmatização dos distúrbios mentais ainda se faz persistente, manifestando-se em um significativo obstáculo para a busca por ajuda e tratamento de qualquer forma de sofrimento psicológico (ELEFTHERIADES et al., 2020). Conforme levantamento de Eisenberg, Golberstein, e Gollust (2007), que buscaram as principais razões pelas quais estudantes que necessitavam de apoio psicoemocional não o buscavam, $20 \%$ admitiram que não procuravam ajuda por receio do que os outros pensariam deles caso soubessem que estavam fazendo algum tipo de tratamento mental. Ainda, deste mesmo total de entrevistados, $32 \%$ alegaram não buscar apoio por falta de tempo diante das atividades acadêmicas, enquanto 8\% alegaram não ter condições financeiras para tal. Tais proporções, conjuntamente com os resultados aqui alcançados, reforçam a preocupação com o estigma ainda presente no ambiente universitário, bem como despertam questionamentos a respeito da estrutura do sistema de educação superior e se ele vem, de fato, oferecendo os meios necessários de apoio e promoção da saúde mental na esfera acadêmica. A confirmação empírica de tais conjecturas deve, no entanto, ser estendida para trabalhos futuros.

\section{Conclusão}

Estudos recentes revelam que níveis educacionais mais elevados tendem a levar ao desenvolvimento de distúrbios psicoemocionais. Diante do crescimento da quantidade de pós-graduandos brasileiros, bem como das taxas de prevalência de transtornos mentais comuns no Brasil, surgiu o propósito de observar os custos psicológicos por um alto nível de educação. Por meio da abordagem do Propensity Score Matching, o presente estudo buscou estimar o impacto da pós-graduação stricto sensu (mestrado e doutorado) no estado de saúde mental dos indivíduos - sendo este estado captado pelo diagnóstico de depressão clínica.

Estimados por três técnicas distintas de pareamento - nearest neighbor, radius e kernel matching - os efeitos da alta qualificação sobre a saúde mental se mostraram estatisticamente prejudiciais, revelando que frequentar um curso de pós-graduação stricto sensu exerceu impacto positivo sobre os diagnósticos de depressão clínica e exibindo, portanto, efeito depreciativo sobre o estado de saúde mental das observações analisadas. Quando se fez a mesma análise diferenciando os sexos, foi possível observar que os efeitos da pós-graduação foram nocivos para o público masculino, ao passo que, para as mulheres, não foram estatisticamente significativos.

Diante da escassez de base de dados referentes aos distúrbios mentais enfrentados pela população brasileira, as limitações deste estudo remetem, em última 
instância, ao tamanho relativamente pequeno da amostra tanto de pós-graduados quanto de indivíduos clinicamente depressivos. Atenta-se também para o risco de subnotificação dos diagnósticos de depressão dentro do inquérito utilizado como fonte de informações, especialmente no que se concerne ao público masculino. Por lidarem com um grau mais elevado de estigma em torno da discussão sobre saúde mental, homens acabam sendo menos inclinados a buscar ajuda psicoemocional quando comparados às mulheres. Deve-se, também, reconhecer a limitação metodológica do presente estudo. Por se tratar de um estudo de corte transversal, não houve a possibilidade de se utilizar métodos de estimação - como o método de diferença em diferenças, por exemplo - que observassem e controlassem para os efeitos do tratamento ao longo do tempo.

Não obstante, generalizar para outros países o efeito da pós-graduação aqui obtido pode consistir em um pretensioso erro de pesquisa diante das especificidades sociais, econômicas, políticas e culturais de cada espaço geográfico. Sugere-se, contudo, a promoção do diálogo aberto e esclarecedor sobre saúde mental no ambiente acadêmico, a fim de que, deste modo, o debate em si contribua como medida de prevenção a favor da plenitude psicológica do capital humano brasileiro.

\section{Esclarecimentos}

O presente trabalho foi realizado com apoio da Coordenação de Aperfeiçoamento de Pessoal de Nível Superior - Brasil (CAPES) - Código de Financiamento 001.

\section{Referências}

ADEWUYA, A.O. Depression amongst Nigerian University Students. Social Psychiatry and Psychiatric Epidemiology, v. 41, p. 674-678, 2006.

DOI: https://doi.org/10.1007/s00127-006-0068-9

AL-SHAYEA, E. I. Perceived Depression, Anxiety and Stress among Saudi Postgraduate Orthodontic Students: a multi-institutional survey. Pakistan Oral \& Dental Journal, v. 34, n. 2, p. 296-303, 2014.

https://www.thefreelibrary.com/Perceived+depression\%2c+anxiety+and+stress+among+ Saudi+postgraduate...-a0381474444.

AUSTIN, P. C. Propensity Score Matching and Complex Surveys. Statistical Methods in Medical Research, v. 27, n. 4, p. 1240-1257, 2016.

DOI: https://doi.org/10.1177/0962280216658920

AUSTIN, P. C.; SMALL, D. S. The Use of Bootstrapping when using Propensity-Score Matching without Replacement: A simulation study. Statistics in Medicine, v. 33, n. 24, p. 4306-4319, 2014. DOI: https://doi.org/10.1002/sim.6276 
BAYRAM, N.; BILGEL, N. The Prevalence and Socio-Demographic Correlations of Depression, Anxiety and Stress among a Group of University Students. Social Psychiatry and Psychiatric Epidemiology. v. 43, n. 8, p. 667-672, 2008.

DOI: https://doi.org/10.1007/s00127-008-0345-X

BENJET, C. Youth Mental Health in a Populous City of the Developing World: Results from the Mexican Adolescent Mental Health Survey. Journal of Child Psychology and Psychiatry, v. 50, n. 4, p. 386-395, 2009.

DOI: https://doi.org/10.1111/j.1469-7610.2008.01962.x

BISHOP, J. B. The Changing Student Culture: Implications for counselors and administrators. Journal of College Student Psychotherapy, v. 6, n. 3-4, p. 37-57, 1993. DOI: https://doi.org/10.1300/J035v06n03_03

BOYD, A. Gender Differences in Mental Disorders and Suicidality in Europe: Results from a large cross-sectional population-based study. Journal of Affective Disorders, v. 173, p. 245-254, 2015. DOI: https://doi.org/10.1016/j.jad.2014.11.002

BRASIL. Ministério do Planejamento, Orçamento e Gestão. Instituto Brasileiro de Geografia e Estatística. Diretoria de Pesquisas. Pesquisa Nacional de Saúde: percepção do estado de saúde, estilos de vida e doenças crônicas. Rio de Janeiro: IBGE, 2014. 181p.

BRASIL. Ministério do Planejamento, Orçamento e Gestão. Instituto Brasileiro de Geografia e Estatística. Diretoria de Pesquisas. Pesquisa Nacional por Amostra de Domicílios. Rio de Janeiro: IBGE, 2015.

\section{BRASIL. Qual a Diferença entre Pós-Graduação Lato Sensu e Stricto Sensu?} Brasília, DF. 2018. Disponível em:

<http://portal.mec.gov.br/component/content/article?id=13072:qual-a-diferenca-entrepos-graduacao-lato-sensu-e-stricto-sensu>. Acesso em: 6 de jul. 2020.

CALIENDO, M.; KOPEINIG, S. Some Practical Guidance for the Implementation of Propensity Score Matching. The Institute for the Study of Labor (IZA), v. 1588, p. 132, 2005. DOI: https://doi.org/10.1111/j.1467-6419.2007.00527.x

CICIURKAITE, G.; PERRY, B. L. Body Weight, Perceived Weight Stigma and Mental Health among Women at the Intersection of Race/Ethnicity and Socioeconomic Status: Insights from the modified labelling approach. Sociology of Health and Illness, v. 40, n. 1, p. 18-37, 2018. DOI: https://doi.org/10.1111/1467-9566.12619

CIRANI, C. B. S. A Evolução do Ensino da Pós-Graduação Senso Estrito no Brasil: Análise exploratória e proposições para pesquisa. Avaliação: Revista da Avaliação da Educação Superior (Campinas), v. 20, n. 1, p. 163-187, 2015.

DOI: https://doi.org/10.590/S1414-40772015000500011 
COORDENAÇÃO DE APERFEIÇOAMENTO DE PESSOAL DE NÍVEL SUPERIOR. CAPES - [2017 a 2020] Sucupira: Coleta de Dados, Discentes da Pós-Graduação stricto sensu do Brasil. Brasília: CAPES, 2019a.

COORDENAÇÃO DE APERFEIÇOAMENTO DE PESSOAL DE NÍVEL SUPERIOR. CAPES - CAPES: Coleta de Dados, Discentes dos Programas de Pós-Graduação stricto sensu no Brasil 2004 a 2012. Brasília: CAPES, 2019b.

COORDENAÇÃO DE APERFEIÇOAMENTO DE PESSOAL DE NÍVEL SUPERIOR. CAPES - Sucupira: Coleta de Dados, Discentes da Pós-Graduação stricto sensu do Brasil 2013 a 2016. Brasília: CAPES, 2018.

COUGHLAN, S. Rising Numbers of Stressed Students Seek Help. 2015. Disponível em: <https://www.bbc.com/news/education-34354405>. Acesso em: 8 de jul. 2020.

COSTA, E. G.; NEBEL, L. O Quanto Vale a Dor? Estudo sobre a saúde mental de estudantes de pós-graduação no Brasil. Polis, v. 50, p. 1-20, 2018.

DOI: http://dx.doi.org/10.4067/S0718-65682018000200207

CUONG, N. V. Selection of Control Variables in Propensity Score Matching: evidence from a simulation study. Munich Personal RePEc Archive, p. 1-15, 2012.

https://mpra.ub.uni-muenchen.de/36377/.

EISENBERG, D.; GOLLUST, S.E.; GOLBERSTEIN, E. Prevalence and Correlates of Depression, Anxiety, and Suicidality among University Students. American Journal of OrthopsyChiatry, v. 77, n. 4, p. 534-542, 2007.

DOI: http://dx.doi.org/10.1037/0002-9432.77.4.534

ELEFTHERIADES, R. The Challenges and Mental Health Issues of Academic Trainees.

F1000 Research, v. 9, n. 104, p. 1-26, 2020.

DOI: http://dx.doi.org/10.12688/f1000research.21066.1

ELSTAD, J. I.; PEDERSEN, A. W. The Impact of Relative Poverty on Norwegian Adolescents' Subjective Health: A Causal Analysis with Propensity Score Matching. International Journal of Environmental Research and Public Health. v. 9, p. 47154731, 2012. DOI: http://dx.doi.org/10.3390/ijerph9124715

EVANS, T. M. More than One-Third of Graduate Students Report Being Depressed. International Journal of Science. v. 36, p. 282-284, 2018.

DOI: https://doi.org/10.1038/d41586-018-03803-3

FANG, J. Depression Prevalence in Postgraduate Students and its Association with GAIT Abnormality. IEEE Access, vol. 7, p. 174425-174437, 2019.

DOI: https://doi.org/10.1109/ACCESS.2019.2957179

FARRER, L. M. Demographic and Psychosocial Predictors of Major Depression and Generalized Anxiety Disorder in Australian University Students. BMC Psychiatry, v. 16, n. 241, p. 1-9, 2016. DOI: https://doi.org/10.1186/s12888-016-0961-z 
FAYISSA, B.; GUTEMA, P. A Health Production Function for Sub-Saharan Africa (SSA). Applied Economics, v. 37, n. 2, p. 155-164, 2006.

DOI: https://doi.org/10.1080/00036840412331313521

FAYISSA, B.; TRAIAN, A. Estimation of a Health Production Function: Evidence from East European Countries. The American Economist, v. 58, n. 2, p. 134-148, 2013. DOI: https://doi.org/10.1177/056943451305800206.

FEINSTEIN, L. Quantitative Estimates of the Social Benefits of Learning, 2: Health (depression and obesity), wider benefits of learning. Research Report: 6, London: Centre for Research on the Wider Benefits of Learning, Institute of Education, 2002. https://discovery.ucl.ac.uk/id/eprint/10018651

FOLLAND, S.; GOODMAN, A. C.; STANO, M. The Economics of Health and Health Care. 7 ed. Pearson, 2013. 625p.

FOND, G. Psychiatric and Psychological Follow-Up of Undergraduate and Postgraduate Medical Students: Prevalence and associated factors. Results from the national BOURBON study. Psychiatry Research, v. 272, p. 425-430, 2019.

DOI: https://doi.org/10.1016/j.psychres.2018.12.174.

FONTES, L. F. C.; CONCEIÇÃO, O. C.; MACHADO, S. Violência Sexual na Adolescência, Perfil da Vítima e Impactos sobre a Saúde Mental. Ciência \& Saúde Coletiva. v. 22, n. 9, p. 2919-2928, 2017.

DOI: https://doi.org/10.1590/1413-81232017229.11042017

GIORDAN, I. 21\% dos Brasileiros Possuem Ensino Superior Completo, Aponta Levantamento da OCDE. 2019. Disponível em: < https://querobolsa.com.br/revista/21dos-brasileiros-possuem-ensino-superior-completo-aponta-levantamento-da-ocde〉. Acesso em: 5 de jul. 2020.

GLOBAL HEALTH INTELLIGENCE. WHO: Brazil has highest number of people suffering from depression in Latin America. 2017. Disponível em: <http://globalhealthintelligence.com/news/brazil-highest-number-people-sufferingdepression-latin-america/>. Acesso em: 8 jan. 2019.

GROSSMAN, M. The Demand for Health: A theoretical and empirical investigation. New York: Columbia University Press, 1972.

HAGEN, T. Econometric Evaluation of a Placement Coaching Program for Recipients of Disability Insurance Benefits in Switzerland. Working Paper Series: Business and Law, v. 10, p. 1-46, 2016. DOI: https://doi.org/10.13140/RG.2.1.4711.2084

HECKMAN, J. J.; ICHIMURA, H.; TODD, P. Matching as an Econometric Evaluation Estimator. Review of Economic Studies, v. 65, p. 261-294, 1997.

DOI: https://doi.org/10.2307/2971733 
HEIM, C. Pituitary-Adrenal and Autonomic Responses to Stress in Women after Sexual and Physical Abuse in Childhood. Journal of the American Medical Association. v. 284, n. 5, p. 592-597, 2000. DOI: https://doi.org/10.1001/jama.284.5.592

HSU, K.; MARSHALL, V. Prevalence of Depression and Distress in a Large Sample of Canadian Residents, Interns, and Fellows. American Journal of Psychiatry, v. 144, n. 12, p. 1531-1566, 1987. DOI: https://doi.org/10.1176/ajp.144.12.1561

HUANG, P. Fruit and Vegetable Consumption and Mental Health across Adolescence: Evidence from a diverse urban British cohort study. International Journal of Behavioral Nutrition and Physical Activity, v. 16, n. 19, p. 1-13, 2019. DOI: https://doi.org/10.1186/s12966-019-0780-y

IBRAHIM, A. K. A Systematic Review of Studies of Depression Prevalence in University Students. Journal of Psychiatric Research. v. 47, n. 3, p. 391-400, 2013. DOI: https://doi.org/10.1016/j.jpsychires.2012.11.015

JAEN-VARAS, D. The Association Between Adolescent Suicide Rates and Socioeconomic Indicators in Brazil: A 10-year retrospective ecological study. Brazilian Journal of Psychiatry, v. 41, n. 5, p. 389-395, 2019.

DOI: https://doi.org/10.1590/1516-4446-2018-0223

JOHNS, M. M. Resilient Minds and Bodies: Size discrimination, body image, and mental health among sexual minority women. Psychology of Sexual Orientation and Gender Diversity, v. 4, n. 1, p. 34-42, 2017. DOI: https://doi.org/10.1037/sgd0000207

KASSOUF, A. L. Função de Produção de Saúde em Diferentes Regiões e Setores do Brasil. Pesquisa e Planejamento Econômico, v. 23, n 3, p. 547-570, 1993.

KENKEL, D. S. Should you Eat Breakfast? Estimates from health production functions. Health Economics, v. 4, n. 1, p. 15-29, 1995.

DOI: https://doi.org/10.1002/hec.4730040103

KHANDER, S. R.; KOOLWAL, G. B.; SAMAD, H. A. Handbook on Impact Evaluation. 1 ed. Washington, D. C.: World Bank. 2010. Cap 4.

KHUSHDE, S. A Comparative Study of Homesickness, Depression, and Internet Addiction between Native and Nonnative Students at University of Sistan and Baluchestan, Iran. Annals of Tropical Medicine and Public Health, v. 10, p. 15371546, 2017. DOI: https://doi.org/10.4103/ATMPH.ATMPH_498_17

KNAPP, M. Primary Prevention of Child Mental Health Problems using Primary Health Care Professionals: Cost comparisons. International Journal of Mental Health Promotion, v. 7, n. 1, p. 95-102, 2005.

DOI: https://doi.org/10.1080/14623730.2005.9721953.

KNESPER, D. Preliminary Production Functions Describing Change. Medical Care, v. 1, p. 222-237, 1987. 
KUEHNEN, C. Why is Depression More Common among Women than among Men?

The Lancet Psychiatry, v. 4, n. 2, p. 146-158, 2017.

DOI: https://doi.org/10.1016/S2215-0366(16)30263-2.

LEVECQUE, K. Work Organization and Mental Health Problems in PhD Students.

Research Policy, v. 46, n. 4, p. 868-879, 2017.

DOI: https://doi.org/10.1016/j.respol.2017.02.008

MAGALHÃES, U. Demanda por Saúde no Brasil: Dois estudos de caso. Revista

Brasileira de Economia, v. 32, n. 4, p. 517-556, 1978. http://hdl.handle.net/10438/13067

MARÉS, C. Instituições Públicas Oferecem $80 \%$ dos Programas de Pós-Graduação do País. 2019. Disponível em:

<https://piaui.folha.uol.com.br/lupa/2019/05/16/universidades-federais-pos-

graduacao/\#: :text=Segundo\%20a\%20Capes\%2C\%20o\%20Brasil,\%2C8\%25\%20no\%20 mestrado\%20profissional.> Acesso em: 5 de jul. 2020.

MEIER, S. M. Attention-Deficit Hyperactivity Disorder and Anxiety Disorders as Precursors of Bipolar Disorder onset in Adulthood. The British Journal of Psychiatry, v. 213, p. 555-560, 2018. DOI: https://doi.org/10.1192/bjp.2018.111

NISBET, R. Model Evaluation and Enhancement. In: NISBET, R.; MINER, G.; YALE, K. Handbook of Statistical Analysis and Data Mining Applications. 2 ed. Amsterdã: Elsevier, 2018. Cap. 11.

NIXON, J.; ULMANN, P. The Relationship between Health Care Expenditures and Health Outcomes. European Journal of Health Economics, v. 7, p. 7-18, 2006.

DOI: https://doi.org/10.1007/s10198-005-0336-8

OHRNBERGER, J. The Relationship between Physical and Mental Health: A mediation analysis. Social Science \& Medicine, v. 195, p. 42-49, 2017.

DOI: https://doi.org/10.1016/j.socscimed.2017.11.008

ORGANISATION FOR ECONOMIC CO-OPERATION AND DEVELOPMENT. Education at a Glance 2019: OECD Indicators. Paris: OECD Publishing, 2019.

ORGANIZAÇÃO PAN-AMERICANA DA SAÚDE. "Suicídio é Grave Problema de Saúde Pública e sua Prevenção deve ser Prioridade”, afirma OPAS/OMS. 2018.

Disponível em:

<https://www.paho.org/bra/index.php?option=com_content\&view=article\&id=5674:suici dio-e-grave-problema-de-saude-publica-e-sua-prevencao-deve-ser-prioridade-afirmaopas-oms\&Itemid=839>. Acesso em: 5 de jul. 2020.

PELUSO, D.L. Depression Symptoms in Canadian Psychology Graduate Students: Do research productivity, funding, and the academic advisory relationship play a role?

Canadian Journal of Behavioural Science, v. 43, p.119-127, 2011.

DOI: https://doi.org/10.1037/A0022624 
PHELPS, C. E. Health Economics. 5 ed. Routledge, 2016. 526p.

RIECHER-RÖSSLER, A. Prospects for the Classification of Mental Disorders in Women. European Psychiatry, v. 25, n. 4, p. 189-196, 2010.

DOI: https://doi.org/10.1016/j.eurpsy.2009.03.002

ROSENBAUM, P. R.; RUBIN, D. B. The Central Role of the Propensity Score in Observational Studies for Causal Effects. Biometrika, v. 70, n. 1, p. 41-55, 1983. DOI: https://doi.org/10.1093/biomet/70.1.41

RUBIN, D.B. Using Propensity Scores to Help Design Observational Studies: Application to the tobacco litigation. Health Services \& Outcomes Research Methodology, v. 2, p. 169-188, 2001. DOI: https://doi.org/10.1023/A:1020363010465

SALAGRE, E. et al. Toward Precision Psychiatry in Bipolar Disorder: Staging 2.0. Frontiers in Psychiatry, v. 9, n. 641, p. 1-15, 2018.

DOI: https://doi.org/10.3389/fpsyt.2018.00641

SANTOS, M. J.; KASSOUF, A. L. Uma Investigação dos Determinantes

Socioeconômicos da Depressão Mental no Brasil com Ênfase nos Efeitos da Educação. Economia Aplicada. v. 11, n. 1, p. 5-26, 2007.

DOI: https://doi.org/10.1590/S1413-80502007000100001

SASSARINI, J. Depression in Midlife Women. Maturitas, v. 94, p. 149-154, 2016. DOI: https://doi.org/10.1016/j.maturitas.2016.09.004.

SORIANO, J. J. Seasonal Variations in Mood and Behavior in Romanian Postgraduate Students. The Scientific World Journal, v. 7, p. 870-879, 2007.

DOI: https://doi.org/10.1100/tsw.2007.127

SOUZA, M. B. C. A Colapso na Academia? O comportamento de pós-graduandos em Administração e o Burnout. Cadernos de Estudos Sociais, v. 33, n. 1, p. 59-84, 2018.

SOUZA, M. C. S. C. Escores de Propensão: Aplicações à Epidemiologia. 2010. Monografia (Graduação em Estatística) - Universidade Federal do Rio Grande do Sul, Porto Alegre, 2010.

STATACORP. Stata: Release 13. College Station, TX: StataCorp LP. 2013.

STORDAL, E.; MYKLETUN, A.; DAHL, A. A. The Association between Age and Depression in the General Population: A multivariate examination. Acta Psychiatrica Scandinavica. v. 107, p. 132-141, 2003.

DOI: https://doi.org/10.1034/j.1600-0447.2003.02056.x

VAN DER WAERDEN, J. E. B. Defining Subgroups of Low Socioeconomic Status Women at Risk for Depressive Symptoms: The importance of perceived stress and cumulative risks. International Journal of Social Psychiatry, v. 60, n. 8, p. 772-782, 2014. DOI: https://doi.org/10.1177/0020764014522751. 
VAN DE VELDE, S.; BRACKE, P.; LEVECQUE, K. Gender Differences in Depression in 23 European Countries. Cross-national variation in the gender gap in depression.

Social Science \& Medicine. v. 71, p. 305-313, 2010.

DOI: https://doi.org/10.1016/j.socscimed.2010.03.035

VÁZQUEZ, F. L.; BLANCO, V. B. Prevalence of DSM-IV Major Depression among Spanish University Students. Journal of American College Health, v. 57, n. 2, p. 165172, 2010. DOI: https://doi.org/10.3200/JACH.57.2.165-172

THE WORLD HEALTH ORGANIZATION. Mental Health: New understanding, new hope: World Health Organization, Genebra. 2001. Disponível em:

<https://www.who.int/whr/2001/en/>. Acesso em: 20 dez. 2018.

WITTCHEN, H. U. The Size and Burden of Mental Disorders and other Disorders of the Brain in Europe. European Neuropsychopharmacology, v. 21, n. 9, p. 655-679, 2011. DOI: https://doi.org/10.1016/j.euroneuro.2011.07.018.

ZANUTTO, E. L. A Comparison of Propensity Score and Linear Regression Analysis of Complex Survey Data. Journal of Data Science, v. 4, p. 67-91, 2006.

DOI: https://doi.org/10.6339/JDS.2006.04(1).233.

ZIMMERMAN, M. Understanding the Severity of Depression: Which symptoms of depression are the best indicators of depression severity? Comprehensive Psychiatry, v. 87, p. 84-88, 2018. DOI: https://doi.org/10.1017/S1092852919001548.

\section{Apêndice}

\section{Tabela 1 - Modelo Probit (modelo de tratamento) Estimado no Processo de Pareamento entre Tratados e não Tratados}

\begin{tabular}{|c|c|c|c|}
\hline \multirow{2}{*}{$\begin{array}{l}\text { Variáveis } \\
\text { independentes }\end{array}$} & \multicolumn{3}{|c|}{ Coeficientes } \\
\hline & Geral & Homem & Mulher \\
\hline Sexo & $-0,015$ & & \\
\hline Raça & $0,515 * * *$ & $0,507 * * *$ & $0,530 * * *$ \\
\hline Idade & $-0,102 * *$ & $-0,100$ & $-0,089$ \\
\hline Ocupação & $0,471 * * *$ & $0,306^{* * *}$ & $0,566^{* * *}$ \\
\hline Constante & $-2,867 * * *$ & $-2,721 * * *$ & $-2,970 * * *$ \\
\hline Log likelihood & $-2.567,12$ & $-1.274,81$ & $-1.287,87$ \\
\hline Teste chi-quadrado & $348,10 * * *$ & $124,05 * * *$ & 223,36 **** \\
\hline Pseudo-R $^{2}$ & 0,063 & 0,046 & 0,079 \\
\hline $\mathbf{N}$ observações & 50.103 & 21.627 & 28.476 \\
\hline
\end{tabular}

Fonte: Elaboração própria (2021).

Nota: $* * *$ Significativo a $1 \%$. **Significativo a $5 \%$. 
Tabela 2 - Balanceamento (redução de viés) das Covariáveis Antes e Depois do Pareamento (teste t)

\begin{tabular}{|c|c|c|c|c|c|c|}
\hline \multirow{3}{*}{\multicolumn{2}{|c|}{$\begin{array}{c}\text { Covariável antes e } \\
\text { depois do } \\
\text { pareamento }\end{array}$}} & \multicolumn{5}{|c|}{ Geral } \\
\hline & & \multicolumn{2}{|c|}{ Média } & \multirow{2}{*}{ Diferença \% (viés) } & \multicolumn{2}{|c|}{ Teste $\mathbf{t}$} \\
\hline & & Tratados & Controle & & $\mathbf{t}$ & p-valor \\
\hline \multirow{2}{*}{ Sexo } & Antes & 0,498 & 0,569 & $-14,1$ & $-3,11$ & 0,002 \\
\hline & Depois & 0,498 & 0,498 & 0,00 & 0,00 & 1,000 \\
\hline \multirow{2}{*}{ Idade } & Antes & 0,821 & 0,833 & 4,6 & 0,99 & 0,321 \\
\hline & Depois & 0,821 & 0,821 & 0,00 & 0,00 & 1,000 \\
\hline \multirow{2}{*}{ Raça } & Antes & 0,739 & 0,406 & 71,3 & 14,87 & \\
\hline & Depois & 0,739 & 0,739 & 0,00 & 0,00 & 1,000 \\
\hline \multirow{5}{*}{ Ocupação } & Antes & 0,825 & 0,584 & 54,8 & 10,77 & 0,000 \\
\hline & Depois & 0,825 & 0,825 & 0,00 & 0,00 & 1,000 \\
\hline & & \multicolumn{5}{|c|}{ Homem } \\
\hline & & \multicolumn{2}{|c|}{ Média } & \multirow{2}{*}{ Diferença \% (viés) } & \multicolumn{2}{|c|}{ Teste $t$} \\
\hline & & Tratados & Controle & & $t$ & p-valor \\
\hline \multirow{2}{*}{ Idade } & Antes & 0,803 & 0,812 & $-2,3$ & $-0,36$ & 0,718 \\
\hline & Depois & 0,803 & 0,803 & 0,00 & 0,00 & 1,000 \\
\hline \multirow{2}{*}{ Raça } & Antes & 0,725 & 0,400 & 69,3 & 10,32 & 0,000 \\
\hline & Depois & 0,725 & 0,725 & 0,00 & 0,00 & 1,000 \\
\hline \multirow{2}{*}{ Ocupação } & Antes & 0,856 & 0,742 & 28,8 & 4,06 & 0,000 \\
\hline & Depois & 0,856 & 0,856 & 0,00 & 0,00 & 1,000 \\
\hline & & \multicolumn{5}{|c|}{ Mulher } \\
\hline & & \multicolumn{2}{|c|}{ Média } & \multirow{2}{*}{ Diferença \% (viés) } & \multicolumn{2}{|c|}{ Teste $t$} \\
\hline & & Tratados & Controle & & $\mathbf{t}$ & p-valor \\
\hline \multirow{2}{*}{ Idade } & Antes & 0,839 & 0,796 & 11,1 & 1,66 & 0,098 \\
\hline & Depois & 0,839 & 0,839 & 0,00 & 0,00 & 1,000 \\
\hline \multirow{2}{*}{ Raça } & Antes & 0,753 & 0,411 & 73,7 & 10,77 & 0,000 \\
\hline & Depois & 0,753 & 0,753 & 0,00 & 0,00 & 1,000 \\
\hline \multirow{2}{*}{ Ocupação } & Antes & 0,794 & 0,464 & 72,6 & 10,28 & 0,000 \\
\hline & Depois & 0,794 & 0,794 & 0,00 & 0,00 & 1,000 \\
\hline
\end{tabular}

Fonte: Elaboração própria (2021).

Nota: Um p-valor maior que 0,10 indica que a diferença entre tratados e controle não é significativa, de maneira que as covariáveis estejam sendo observadas em grupos comparáveis. 